\title{
Exponential Synchronization of Impulsive Complex Networks with Output Coupling
}

\author{
Yue-Hui Zhao $^{1} \quad$ Jin-Liang Wang ${ }^{2}$ \\ ${ }^{1}$ College of Post and Telecommunication of Wuhan Institute of Technology, Wuhan 430070, China \\ ${ }^{2}$ Science and Technology on Aircraft Control Laboratory, School of Automation Science and Electrical Engineering, Beihang University, \\ Beijing 100191, China
}

\begin{abstract}
This paper proposes a new impulsive complex delayed dynamical network model with output coupling, which is totally different from some existing network models. Then, by employing impulsive delay differential inequalities, some sufficient conditions are obtained to guarantee the global exponential state synchronization and output synchronization of the impulsive complex delayed dynamical network. Finally, two numerical examples are given to demonstrate the effectiveness of the obtained results.
\end{abstract}

Keywords: Impulsive complex networks, output coupling, state synchronization, output synchronization, impulsive delay differential inequalities.

\section{Introduction}

In the past ten years, there have been many researchers studying the topology and dynamical behavior of complex networks. The main reason is that many practical systems can be characterized by various models of complex networks. Some well-known examples include food webs, communication networks, social networks, power grids, cellular networks, world wide web, metabolic systems, disease transmission networks, etc. ${ }^{[1,2]}$ In particular, the synchronization problem has received much of the focus in recent years. A wide variety of synchronization criteria have been presented for various complex networks ${ }^{[3-25]}$. Wang et al. ${ }^{[4]}$ considered a generalized complex network possessing general topology. Some criteria on local and global exponential synchronization were derived by constructing suitable Lyapunov functionals. Li and Lai ${ }^{[19]}$ studied the adaptiveimpulsive synchronization of uncertain complex networks. Based on the stability analysis of impulsive system, several synchronization criteria for local and global adaptiveimpulsive synchronization were established. But, it should be noticed that the complex networks with state coupling were considered in these articles.

Jiang et al. ${ }^{[26]}$ first introduced a complex dynamical network model with output coupling without time delays. Practically, the time delays always exist in complex networks due to the finite speeds of transmission and/or the traffic congestion, and most of the delays are notable. Moreover, in practical evolutionary processes of the networks, absolute constant delay may be scarce and delays are frequently varying with time. Unfortunately, only a few authors have considered the synchronization for complex networks with time-varying delay and output coupling ${ }^{[27]}$. Therefore, it is essential to further study the synchroniza-

Manuscript received June 8, 2012; revised October 22, 2012

This work was supported by Key Project of Chinese Education Ministry (No.212138), Natural Science Foundation of Chongqing (No. CQ CSTC 2011BB0117), Foundation of Science and Technology Project of Chongqing Education Commission (No. KJ120630), Innovation Foundation of BUAA for PhD Graduates (No. YWF-12RBYJ-005). tion of complex dynamical networks with time-varying delay and output coupling.

Recently, many researchers have studied the stability of impulsive systems ${ }^{[28-36]}$. Yang and $\mathrm{Xu}^{[29]}$ investigated a class of impulsive control systems with time-varying delays. They analyzed the global exponential stability of the impulsive delay systems and estimated the exponential convergence rate. Yang and $\mathrm{CaO}^{[35]}$ considered the exponential synchronization of complex dynamical network with a coupling delay and impulses. Several criteria for exponential synchronization were derived by the geometrical decomposition of network states and linear matrix inequality method. To the best of our knowledge, the state synchronization of impulsive complex delayed networks with output coupling has not yet been established.

State synchronization is an important direction in the complex networks. However, the node state in complex networks is difficult to be observed or measured, even the node state cannot be observed or measured at all. Moreover, in many circumstances, only part states are needed to make the synchronization to come true ${ }^{[27]}$. Therefore, it is very important and interesting to study the output synchronization of complex networks.

Motivated by the above discussions, a new impulsive complex delayed dynamical network model with output coupling is proposed. The objective of this paper is to study the state synchronization and output synchronization of the proposed network model. Some sufficient conditions ensuring the state synchronization and output synchronization are obtained by using impulsive delay differential inequalities.

The rest of this paper is organized as follows. In Section 2, our mathematical model of the complex network is presented and some preliminaries are given. Several state synchronization and output synchronization criteria are established in Section 3. In Section 4, numerical examples are given to illustrate the effectiveness of the proposed results. Concluding remarks are collected in Section 5. 


\section{Network model and preliminaries}

Let $\mathbf{R}^{+}=[0,+\infty), \mathbf{N}=\{1,2,3, \cdots\}, \mathbf{R}^{n}$ be the $n$ dimensional Euclidean space, and $\mathbf{R}^{n \times m}$ be the space of $n \times m$ real matrices. $P \in \mathbf{R}^{n \times n}>0\left(P \in \mathbf{R}^{n \times n}<0\right)$ means that matrix $P$ is symmetric and positive (negative) definite. $I_{n}$ denotes the $n \times n$ real identity matrix. $\lambda_{m}(\cdot)$ and $\lambda_{M}(\cdot)$ denote the minimum and the maximum eigenvalues of the corresponding matrix, respectively. $B^{\mathrm{T}}$ denotes the transpose of a square matrix $B$. $\|\cdot\|$ denotes the Euclidean norm for vector or the spectral norm of matrix. $C\left([-\tau, 0], \mathbf{R}^{n}\right)$ is a Banach space of continuous functions mapping the interval $[-\tau, 0]$ into $\mathbf{R}^{n}$ with the norm $\|\phi\|_{\tau}=$ $\sup _{-\tau \leqslant \theta \leqslant 0}\|\phi(\theta)\| . P C[I, \Omega]=\{\phi:[-\tau,+\infty) \rightarrow \mathbf{R}, \phi(t)$ is continuous everywhere except for the points $t_{k}(k \in \mathbf{N})$ at which $\phi\left(t_{k}^{+}\right)=\phi\left(t_{k}\right)$ and $\phi\left(t_{k}^{-}\right)$exists $\}$.

Throughout this paper, we consider an impulsive complex delayed dynamical network consisting of $N$ identical nodes with diffusive and output coupling. The mathematical model of the coupled network can be described as

$$
\left\{\begin{aligned}
\dot{x}_{i}(t)= & f\left(x_{i}(t)\right)+\frac{a}{k_{i}^{\beta_{\omega}}} \sum_{j=1}^{N} L_{i j} \Gamma y_{j}(t-\tau(t)), \\
& t \neq t_{k}, \quad t \geqslant 0 \\
y_{i}(t)= & C x_{i}(t) \\
\Delta x_{i}= & x_{i}\left(t_{k}^{+}\right)-x_{i}\left(t_{k}^{-}\right)=B_{i k} x_{i}\left(t_{k}^{-}\right), \\
& t=t_{k}, \quad k \in \mathbf{N}
\end{aligned}\right.
$$

where $i=1,2, \cdots, N$, and $\tau(t)$ is the time-varying delay with $0 \leqslant \tau(t) \leqslant \tau$.

The function $f(\cdot)$, describing the local dynamics of the nodes, is continuously differentiable and capable of producing various rich dynamical behaviors. $x_{i}(t)=$ $\left(x_{i 1}(t), x_{i 2}(t), \cdots, x_{i n}(t)\right)^{\mathrm{T}} \in \mathbf{R}^{n}$ is the state variable of node $i, y_{i}(t) \in \mathbf{R}^{q}(1 \leqslant q \leqslant n)$ is the output of node $i, C$ and $B_{i k}$ are known matrices with appropriate dimensions, $\Gamma \in \mathbf{R}^{n \times q}$ is inner-coupling matrix which describes the individual coupling between two connected nodes of the network, $a$ is a positive real number, which represents the overall coupling strength, the fixed moments $t_{k}$ satisfy $0=t_{0}<t_{1}<t_{2}<\cdots, \lim _{k \rightarrow+\infty} t_{k}=+\infty, k \in \mathbf{N}, k_{i}$ is the degree of node $i, \beta_{\omega}$ is a tunable weight parameter, the real matrix $L=\left(L_{i j}\right)_{N \times N}$ is a symmetric matrix with diagonal entries $L_{i i}=-k_{i}$ and off-diagonal entries $L_{i j}=1$ if node $i$ and node $j$ are connected by a link, and $L_{i j}=0$ otherwise.

In this paper, we always assume that complex network (1) is connected, and $x_{i}(t)$ is right continuous at $t=t_{k}$, i.e., $x_{i}\left(t_{k}\right)=x_{i}\left(t_{k}^{+}\right)$. Let $x(t)=\left(x_{1}^{\mathrm{T}}(t), x_{2}^{\mathrm{T}}(t), \cdots, x_{N}^{\mathrm{T}}(t)\right)^{\mathrm{T}}$, $y(t)=\left(y_{1}^{\mathrm{T}}(t), y_{2}^{\mathrm{T}}(t), \cdots, y_{N}^{\mathrm{T}}(t)\right)^{\mathrm{T}}$. The initial condition associated with the network (1) is given in the form:

$$
\begin{aligned}
x(s) & =\Phi(s), s \in[-\tau, 0], \phi_{i} \in C\left([-\tau, 0], \mathbf{R}^{n}\right) \\
\Phi(s) & =\left(\phi_{1}^{\mathrm{T}}(s), \phi_{2}^{\mathrm{T}}(s), \cdots, \phi_{N}^{\mathrm{T}}(s)\right)^{\mathrm{T}} \\
y(s) & =\operatorname{diag}(C, C, \cdots, C) \Phi(s), s \in[-\tau, 0] .
\end{aligned}
$$

Next, we give several useful definitions and lemmas.

Definition 1. The complex network (1) is said to achieve state synchronization if

$$
\lim _{t \rightarrow+\infty}\left\|x_{i}(t)-x_{j}(t)\right\|=0, \text { for all } i, j=1,2, \cdots, N .
$$

Definition 2. ${ }^{[27]}$ The complex network (1) is said to achieve output synchronization if

$$
\lim _{t \rightarrow+\infty}\left\|y_{i}(t)-y_{j}(t)\right\|=0, \text { for all } i, j=1,2, \cdots, N .
$$

Definition 3. (see [1]) Let $A=\left(a_{i j}\right)_{m \times n} \in \mathbf{R}^{m \times n}$ and $B=\left(b_{i j}\right)_{p \times q} \in \mathbf{R}^{p \times q}$. Then the Kronecker product (or tensor product) of $A$ and $B$ is defined as

$A \otimes B=\left(\begin{array}{cccc}a_{11} B & a_{12} B & \cdots & a_{1 n} B \\ a_{21} B & a_{22} B & \cdots & a_{2 n} B \\ \cdots & & \cdots & \cdots \\ a_{m 1} B & a_{m 2} B & \cdots & a_{m n} B\end{array}\right) \in \mathbf{R}^{m p \times n q}$.

The Kronecker product has the following properties:

$$
\begin{array}{ll}
\text { 1) } & (A \otimes B)^{\mathrm{T}}=A^{\mathrm{T}} \otimes B^{\mathrm{T}} \\
\text { 2) } & (\alpha A) \otimes B=A \otimes(\alpha B) \\
\text { 3) } & (A+B) \otimes C=A \otimes C+B \otimes C \\
\text { 4) } & (A \otimes B)(C \otimes D)=(A C) \otimes(B D)
\end{array}
$$

where $\alpha \in \mathbf{R}, C, D$ are matrices with suitable dimensions.

Lemma 1. (see [5]) Let $0 \leqslant \tau_{i}(t) \leqslant \tau, i=1,2, \cdots, m$. If there exist real numbers $\sigma>0, \hat{\vartheta}_{1} \geqslant 0, \hat{\vartheta}_{2} \geqslant 0, \cdots, \hat{\vartheta}_{m} \geqslant 0$ and $\vartheta$ such that

$$
\left\{\begin{aligned}
D^{+} u(t) \leqslant & \vartheta u(t)+\hat{\vartheta}_{1} u\left(t-\tau_{1}(t)\right)+\hat{\vartheta}_{2} u\left(t-\tau_{2}(t)\right)+\cdots+ \\
& \hat{\vartheta}_{m} u\left(t-\tau_{m}(t)\right), t \geqslant 0 \\
u\left(t_{k}\right) \leqslant & \sigma u\left(t_{k}^{-}\right), \quad k \in \mathbf{N}
\end{aligned}\right.
$$

$$
\left\{\begin{aligned}
D^{+} v(t)> & \vartheta v(t)+\hat{\vartheta}_{1} v\left(t-\tau_{1}(t)\right)+\hat{\vartheta}_{2} v\left(t-\tau_{2}(t)\right)+\cdots+ \\
& \vartheta_{m} v\left(t-\tau_{m}(t)\right), t \geqslant 0 \\
v\left(t_{k}\right)= & \sigma v\left(t_{k}^{-}\right), \quad k \in \mathbf{N}
\end{aligned}\right.
$$

then $u(t) \leqslant v(t)$, for $-\tau \leqslant t \leqslant 0$ implies

$$
u(t) \leqslant v(t), \text { for } t \geqslant 0
$$

where $u(t), v(t) \in P C[-\tau,+\infty), \mathbf{R}]$.

Lemma 2. (see [35]) Suppose $p>q \geqslant 0$ and $u(t)$ satisfies scalar impulsive differential inequality

$$
\left\{\begin{aligned}
D^{+} u(t) & \leqslant-p u(t)+q\left(\sup _{t-\tau \leqslant s \leqslant t} u(s)\right), \quad t \neq t_{k}, \quad t \geqslant 0 \\
u\left(t_{k}\right) & \leqslant a_{k} u\left(t_{k}^{-}\right), \quad k \in \mathbf{N} \\
u(t) & =\phi(t), \quad t \in[-\tau, 0]
\end{aligned}\right.
$$

where $u(t)$ is continuous at $t \neq t_{k}, u\left(t_{k}\right)=u\left(t_{k}^{+}\right)=$ $\lim _{t \rightarrow t_{k}^{+}} u(t)$, and $u\left(t_{k}^{-}\right)=\lim _{t \rightarrow t_{k}^{-}} u(t), \phi \in P C([-\tau, 0], R)$. Then,

$$
u(t) \leqslant\left(\prod_{0<t_{k} \leqslant t} \theta_{k}\right) \mathrm{e}^{-\mu t}\left(\sup _{-\tau \leqslant s \leqslant 0} \phi(s)\right)
$$

where $\theta_{k}=\max \left\{1,\left|a_{k}\right|\right\}$ and $\mu>0$ is a solution of the inequality $\mu-p+q \mathrm{e}^{\mu \tau} \leqslant 0$.

Lemma 3. (see [29]) Let $P \in \mathbf{R}^{n \times n}>0$ and $P=Q^{\mathrm{T}} Q$. For any $x, \bar{x} \in \mathbf{R}^{n}$ and $A \in \mathbf{R}^{n \times n}$
1) $x^{\mathrm{T}} A^{\mathrm{T}} P A x \leqslant\left\|Q A Q^{-1}\right\|^{2} x^{\mathrm{T}} P x$
2) $\left|x^{\mathrm{T}} P \bar{x}\right| \leqslant \sqrt{x^{\mathrm{T} P x}} \sqrt{\bar{x}^{\mathrm{T}} P \bar{x}}$. 


\section{$3 \quad$ Main results}

Lu et al. ${ }^{[37,38]}$ made the assumption that the function $f(\cdot)$ is in the QUAD function class.

Assumption 1. There exist positive definite diagonal matrix $P=\operatorname{diag}\left(p_{1}, p_{2}, \cdots, p_{n}\right)$ and a diagonal matrix $\Delta=$ $\operatorname{diag}\left(\delta_{1}, \delta_{2}, \cdots, \delta_{n}\right)$ such that $f: \mathbf{R}^{n} \rightarrow \mathbf{R}^{n}$ satisfies the following inequality:

$$
(x-y)^{\mathrm{T}} P[f(x)-f(y)-\Delta(x-y)] \leqslant-\eta(x-y)^{\mathrm{T}}(x-y)
$$

for some $\eta>0$ and all $x, y \in \mathbf{R}^{n}$.

It can be verified that Assumption 1 holds for many of the benchmark chaotic systems including the Lorenz system, the Chen system, the Lü system, and the unified chaotic system. In this paper, we always assume $f(0)=0$. If we let $y=0$ in Assumption 1, then we have

Assumption 2. There exists a positive definite diagonal matrix $P=\operatorname{diag}\left(p_{1}, p_{2}, \cdots, p_{n}\right)$ and a diagonal matrix $\Delta=\operatorname{diag}\left(\delta_{1}, \delta_{2}, \cdots, \delta_{n}\right)$ such that $f$ satisfies the following inequality:

$$
x^{\mathrm{T}} P(f(x)-\Delta x) \leqslant-\eta x^{\mathrm{T}} x
$$

for some $\eta>0$ and all $x \in \mathbf{R}^{n}$.

For convenient analysis, we let

$$
\begin{aligned}
G_{i j} & =\frac{L_{i j}}{k_{i}^{\beta_{\omega}}} \\
Q & =P^{\frac{1}{2}}=\operatorname{diag}\left(\sqrt{p_{1}} \sqrt{p_{2}}, \cdots, \sqrt{p_{n}}\right) \\
\hat{P} & =\operatorname{diag}(P, P, \cdots, P) \\
\hat{Q} & =\operatorname{diag}(Q, Q, \cdots, Q) \\
\hat{\Delta} & =\operatorname{diag}(\Delta, \Delta, \cdots, \Delta) \\
B_{k} & =\operatorname{diag}\left(B_{1 k}, B_{2 k}, \cdots, B_{N k}\right) \\
x(t) & =\left(x_{1}^{\mathrm{T}}(t), x_{2}^{\mathrm{T}}(t), \cdots, x_{N}^{\mathrm{T}}(t)\right)^{\mathrm{T}} .
\end{aligned}
$$

$G=\left(G_{i j}\right)_{N \times N}$ is a coupling matrix, accounting for the topology of the complex dynamical network.

Theorem 1. Let Assumption 2 hold.

1) If there exists a positive constant $\beta<1$ such that

$$
\begin{gathered}
\left\|\hat{Q}\left(I_{n N}+B_{k}\right) \hat{Q}^{-1}\right\| \leqslant \beta, \quad k \in \mathbf{N} \\
2 \lambda_{M}\left(-\eta \hat{P}^{-1}+\hat{\Delta}\right)+\frac{a \lambda_{M}\left((G \otimes \Gamma C)^{\mathrm{T}} \hat{P}(G \otimes \Gamma C)\right)}{\beta^{2} \lambda_{m}(\hat{P})}+ \\
a+\frac{2 \ln \beta}{\rho}<0
\end{gathered}
$$

where $\rho=\sup \left\{t_{k}-t_{k-1}, k \in \mathbf{N}\right\}$, then the network (1) achieves global exponential state synchronization in the following sense:

$$
\|x(t)\| \leqslant \beta^{-1} \sqrt{\frac{\lambda_{M}(\hat{P})}{\lambda_{m}(\hat{P})}}\|\Phi\|_{\tau} \mathrm{e}^{\frac{-\lambda t}{2}}, t \geqslant 0
$$

where $\lambda>0$ is a unique solution of

$$
\begin{aligned}
& \lambda+2 \lambda_{M}\left(-\eta \hat{P}^{-1}+\hat{\Delta}\right)+a+\frac{2 \ln \beta}{\rho}+ \\
& \mathrm{e}^{\lambda \tau} \frac{a \lambda_{M}\left((G \otimes \Gamma C)^{T} \hat{P}(G \otimes \Gamma C)\right)}{\beta^{2} \lambda_{m}(\hat{P})}=0 .
\end{aligned}
$$

2) If there exists a positive constant $\beta \geqslant 1$ such that

$$
\begin{aligned}
& \left\|\hat{Q}\left(I_{n N}+B_{k}\right) \hat{Q}^{-1}\right\| \leqslant \beta, \quad k \in \mathbf{N} \\
& 2 \lambda_{M}\left(-\eta \hat{P}^{-1}+\hat{\Delta}\right)+a+\frac{2 \ln \beta}{\rho}+ \\
& \frac{a \beta^{2} \lambda_{M}\left((G \otimes \Gamma C)^{\mathrm{T}} \hat{P}(G \otimes \Gamma C)\right)}{\lambda_{m}(\hat{P})}<0
\end{aligned}
$$

where $\rho=\inf \left\{t_{k}-t_{k-1}, \quad k \in \mathbf{N}\right\}$, then the network (1) achieves global exponential state synchronization in the following sense:

$$
\|x(t)\| \leqslant \beta \sqrt{\frac{\lambda_{M}(\hat{P})}{\lambda_{m}(\hat{P})}}\|\Phi\|_{\tau} \mathrm{e}^{\frac{-\lambda t}{2}}, t \geqslant 0
$$

where $\lambda>0$ is a unique solution of

$$
\begin{aligned}
& \lambda+2 \lambda_{M}\left(-\eta \hat{P}^{-1}+\hat{\Delta}\right)+a+\frac{2 \ln \beta}{\rho}+ \\
& \mathrm{e}^{\lambda \tau} \frac{a \beta^{2} \lambda_{M}\left((G \otimes \Gamma C)^{\mathrm{T}} \hat{P}(G \otimes \Gamma C)\right)}{\lambda_{m}(\hat{P})}=0 .(7)
\end{aligned}
$$

Proof. First, let $s(\lambda)=\lambda+2 \lambda_{M}\left(-\eta \hat{P}^{-1}+\hat{\Delta}\right)+$ $\mathrm{e}^{\lambda \tau} \frac{a \lambda_{M}\left((G \otimes \Gamma C)^{\mathrm{T}} \hat{P}(G \otimes \Gamma C)\right)}{\beta^{2} \lambda_{m}(\hat{P})}+a+\frac{2 \ln \beta}{\rho}$. From (3), we have $s(0)<0$. Moreover, it is obvious that $s(+\infty)>0$ and $s^{\prime}(\lambda)>0$. Using the continuity and the monotonicity of $s(\lambda)$, (4) has a unique solution $\lambda>0$. Similarly, we can easily obtain that (7) also has a unique solution $\lambda>0$. From (1), we can get

$$
\left\{\begin{aligned}
\dot{x}_{i}(t)= & f\left(x_{i}(t)\right)+a \sum_{j=1}^{N} G_{i j} \Gamma y_{j}(t-\tau(t)), \\
& t \neq t_{k}, \quad t \geqslant 0 \\
y_{i}(t)= & C x_{i}(t) \\
\Delta x_{i}= & x_{i}\left(t_{k}^{+}\right)-x_{i}\left(t_{k}^{-}\right)=B_{i k} x_{i}\left(t_{k}^{-}\right), \\
& t=t_{k}, \quad k \in \mathbf{N}
\end{aligned}\right.
$$

where $i=1,2, \cdots, N$. We can rewrite system (8) in a compact form as

$$
\left\{\begin{aligned}
\dot{x}(t)= & F(x(t))+a(G \otimes \Gamma C) x(t-\tau(t)) \\
& t \neq t_{k}, \quad t \geqslant 0 \\
\Delta x= & x\left(t_{k}^{+}\right)-x\left(t_{k}^{-}\right)=B_{k} x\left(t_{k}^{-}\right) \\
& t=t_{k}, \quad k \in \mathbf{N}
\end{aligned}\right.
$$

where

$$
\begin{aligned}
\Delta x & =\left(\left(\Delta x_{1}\right)^{\mathrm{T}},\left(\Delta x_{2}\right)^{\mathrm{T}}, \cdots,\left(\Delta x_{N}\right)^{\mathrm{T}}\right)^{\mathrm{T}} \\
F(x(t)) & =\left(f^{\mathrm{T}}\left(x_{1}(t)\right), f^{\mathrm{T}}\left(x_{2}(t)\right), \cdots, f^{\mathrm{T}}\left(x_{N}(t)\right)\right)^{\mathrm{T}} .
\end{aligned}
$$


Let $V_{1}(t)=x^{\mathrm{T}}(t) \hat{P} x(t)$. By Lemma 3 , we have

$$
\begin{aligned}
& \dot{V}_{1}(t)=2 x^{\mathrm{T}}(t) \hat{P} \dot{x}(t)= \\
& 2 x^{\mathrm{T}}(t) \hat{P} F(x(t))+2 a x^{\mathrm{T}}(t) \hat{P}(G \otimes \Gamma C) x(t-\tau(t)) \leqslant \\
& 2 x^{\mathrm{T}}(t) \hat{P} F(x(t))+2 a \sqrt{x^{\mathrm{T}}(t) \hat{P} x(t)} \times \\
& \sqrt{x^{\mathrm{T}}(t-\tau(t))(G \otimes \Gamma C)^{\mathrm{T}} \hat{P}(G \otimes \Gamma C) x(t-\tau(t))} \leqslant \\
& 2 x^{\mathrm{T}}(t) \hat{P} F(x(t))+2 a \sqrt{x^{\mathrm{T}}(t) \hat{P} x(t)} \times \\
& \sqrt{\lambda_{M}\left((G \otimes \Gamma C)^{\mathrm{T}} \hat{P}(G \otimes \Gamma C)\right)}\|x(t-\tau(t))\| \leqslant \\
& 2 x^{\mathrm{T}}(t) \hat{P} F(x(t))+a V_{1}(t)+ \\
& a \lambda_{M}\left((G \otimes \Gamma C)^{\mathrm{T}} \hat{P}(G \otimes \Gamma C)\right)\|x(t-\tau(t))\|^{2} \leqslant \\
& 2 x^{\mathrm{T}}(t) \hat{P} F(x(t))+a V_{1}(t)+ \\
& \frac{a \lambda_{M}\left((G \otimes \Gamma C)^{\mathrm{T}} \hat{P}(G \otimes \Gamma C)\right)}{\lambda_{m}(\hat{P})} V_{1}(t-\tau(t)), t \neq t_{k} .
\end{aligned}
$$

According to Assumption 2, we can obtain

$$
\begin{aligned}
& x^{\mathrm{T}}(t) \hat{P} F(x(t))= \\
& \sum_{i=1}^{N} x_{i}^{\mathrm{T}}(t) P f\left(x_{i}(t)\right) \leqslant \\
& \sum_{i=1}^{N}\left[-\eta x_{i}^{\mathrm{T}}(t) x_{i}(t)+x_{i}^{\mathrm{T}}(t) P \Delta x_{i}(t)\right]= \\
& \quad x^{\mathrm{T}}(t)\left(-\eta I_{n N}+\hat{P} \hat{\Delta}\right) x(t) .
\end{aligned}
$$

It follows from (10) that

$$
\begin{aligned}
& \dot{V}_{1}(t) \leqslant 2 x^{\mathrm{T}}(t)\left(-\eta I_{n N}+\hat{P} \hat{\Delta}\right) x(t)+a V_{1}(t)+ \\
& \frac{a \lambda_{M}\left((G \otimes \Gamma C)^{\mathrm{T}} \hat{P}(G \otimes \Gamma C)\right)}{\lambda_{m}(\hat{P})} \times V_{1}(t-\tau(t)), t \neq t_{k} .
\end{aligned}
$$

Let $\nu \in \mathbf{R}^{n N} \neq 0, \psi \in \mathbf{R}^{n N}$ and $z=\hat{Q} \nu$. From the definition of matrix measure induced by vector norm ${ }^{[39]}$,

$$
\begin{aligned}
& \frac{\nu^{\mathrm{T}}\left(-\eta I_{n N}+\hat{P} \hat{\Delta}\right) \nu}{\nu^{\mathrm{T}} \hat{P} \nu}= \\
& \frac{z^{\mathrm{T}}\left(-\eta \hat{P}^{-1}+\hat{\Delta}\right) z}{z^{\mathrm{T}} z} \leqslant \\
& \sup _{\psi \neq 0} \frac{\psi^{\mathrm{T}}\left(-\eta \hat{P}^{-1}+\hat{\Delta}\right) \psi}{\psi^{\mathrm{T}} \psi}= \\
& \lambda_{M}\left(-\eta \hat{P}^{-1}+\hat{\Delta}\right)
\end{aligned}
$$

namely

$$
\begin{aligned}
\nu^{\mathrm{T}}\left(-\eta I_{n N}+\hat{P} \hat{\Delta}\right) \nu & \leqslant \lambda_{M}\left(-\eta \hat{P}^{-1}+\hat{\Delta}\right) \nu^{\mathrm{T}} \hat{P} \nu, \\
\nu & \in \mathbf{R}^{n N} \neq 0 .
\end{aligned}
$$

If $\nu=0,(11)$ is obvious. Then, we can obtain

$$
\begin{gathered}
\dot{V}_{1}(t) \leqslant\left[2 \lambda_{M}\left(-\eta \hat{P}^{-1}+\hat{\Delta}\right)+a\right] V_{1}(t)+ \\
\frac{a \lambda_{M}\left((G \otimes \Gamma C)^{\mathrm{T}} \hat{P}(G \otimes \Gamma C)\right)}{\lambda_{m}(\hat{P})} \times \\
V_{1}(t-\tau(t)), t \neq t_{k} .
\end{gathered}
$$

By Lemma 3, we can get

$$
\begin{aligned}
V_{1}\left(t_{k}\right)= & x^{\mathrm{T}}\left(t_{k}^{-}\right)\left(I_{n N}+B_{k}\right)^{\mathrm{T}} \hat{P}\left(I_{n N}+B_{k}\right) x\left(t_{k}^{-}\right) \leqslant \\
& \left\|\hat{Q}\left(I_{n N}+B_{k}\right) \hat{Q}^{-1}\right\|^{2} x^{\mathrm{T}}\left(t_{k}^{-}\right) \hat{P} x\left(t_{k}^{-}\right) \leqslant \\
& \beta^{2} V_{1}\left(t_{k}^{-}\right), \quad k \in \mathbf{N} .
\end{aligned}
$$

For any $\varepsilon>0$, let $\eta(t)$ be a unique solution of impulsive delay system.

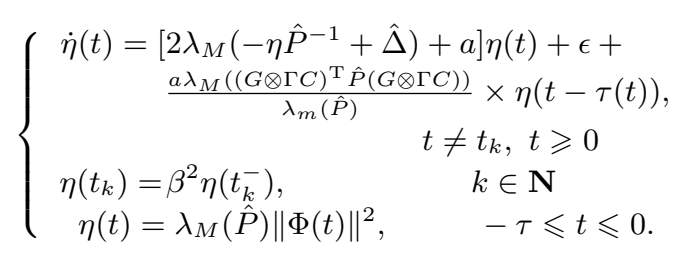

Since $V_{1}(t) \leqslant \lambda_{M}(\hat{P})\|\Phi(t)\|^{2}=\eta(t)$ for $-\tau \leqslant t \leqslant 0$. According to Lemma 1 , we have

$$
\eta(t) \geqslant V_{1}(t) \geqslant 0, \quad t \geqslant 0 .
$$

By the formula for the variation of parameters ${ }^{[40]}$, we have

$$
\begin{aligned}
& \eta(t)=W(t, 0) \eta(0)+ \\
& \int_{0}^{t} W(t, s)\left[\frac{a \lambda_{M}\left((G \otimes \Gamma C)^{T} \hat{P}(G \otimes \Gamma C)\right)}{\lambda_{m}(\hat{P})} \times\right. \\
& \eta(s-\tau(s))+\epsilon] \mathrm{d} s, \quad t \geqslant 0
\end{aligned}
$$

where $W(t, s), t, s \geqslant 0$ is the Cauchy matrix of linear system

$$
\left\{\begin{aligned}
\dot{\varpi}(t) & =\left[2 \lambda_{M}\left(-\eta \hat{P}^{-1}+\hat{\Delta}\right)+a\right] \varpi(t), t \neq t_{k} \\
\varpi\left(t_{k}\right) & =\beta^{2} \varpi\left(t_{k}^{-}\right), \quad k \in \mathbf{N} .
\end{aligned}\right.
$$

Now, we prove the case 1 .

Let $b=-2 \lambda_{M}\left(-\eta \hat{P}^{-1}+\hat{\Delta}\right)-a-\frac{2 \ln \beta}{\rho}$. According to the representation of the Cauchy matrix ${ }^{[40]}$, we get the following estimation since $0<\beta<1$ and $\rho \geqslant t_{k}-t_{k-1}$ :

$$
\begin{aligned}
W(t, s)= & \mathrm{e}^{\left[2 \lambda_{M}\left(-\eta \hat{P}^{-1}+\hat{\Delta}\right)+a\right](t-s)}\left(\prod_{s<t_{k} \leqslant t} \beta^{2}\right) \leqslant \\
& \mathrm{e}^{\left[2 \lambda_{M}\left(-\eta \hat{P}^{-1}+\hat{\Delta}\right)+a\right](t-s)} \beta^{2\left(\frac{t-s}{\rho}-1\right)}= \\
& \mathrm{e}^{\left(-b-\frac{2 \ln \beta}{\rho}\right)(t-s)} \beta^{2\left(\frac{t-s}{\rho}-1\right)}= \\
& \beta^{-2} \mathrm{e}^{-b(t-s)}, \quad t \geqslant s \geqslant 0 .
\end{aligned}
$$

Let $\gamma=\beta^{-2} \lambda_{M}(\hat{P})\|\Phi\|_{\tau}^{2}$. Accordingly

$$
\begin{aligned}
\eta(t) \leqslant & \beta^{-2} \mathrm{e}^{-b t} \lambda_{M}(\hat{P})\|\Phi(0)\|^{2}+ \\
& \int_{0}^{t} \mathrm{e}^{-b(t-s)}\left[\frac{a \lambda_{M}\left((G \otimes \Gamma C)^{T} \hat{P}(G \otimes \Gamma C)\right)}{\beta^{2} \lambda_{m}(\hat{P})} \times\right. \\
& \left.\eta(s-\tau(s))+\frac{\epsilon}{\beta^{2}}\right] \mathrm{d} s \leqslant \\
& \gamma \mathrm{e}^{-b t}+\int_{0}^{t} \mathrm{e}^{-b(t-s)}\left[\frac{a \lambda_{M}\left((G \otimes \Gamma C)^{\mathrm{T}} \hat{P}(G \otimes \Gamma C)\right)}{\beta^{2} \lambda_{m}(\hat{P})} \times\right. \\
& \left.\eta(s-\tau(s))+\frac{\epsilon}{\beta^{2}}\right] \mathrm{d} s, t \geqslant 0 .
\end{aligned}
$$

Since $\epsilon, \lambda>0, b-\frac{a \lambda_{M}\left((G \otimes \Gamma C)^{\mathrm{T}} \hat{P}(G \otimes \Gamma C)\right)}{\beta^{2} \lambda_{m}(\hat{P})}>0$ and $0<\beta<1$, 
we have

$$
\begin{aligned}
\eta(t) & \leqslant \frac{\lambda_{M}(\hat{P})\|\Phi(t)\|^{2}}{\beta^{2}}< \\
& \gamma \mathrm{e}^{-\lambda t}+\frac{\epsilon}{\left[b-\frac{a \lambda_{M}\left((G \otimes \Gamma C)^{\mathrm{T}} \hat{P}(G \otimes \Gamma C)\right)}{\beta^{2} \lambda_{m}(\hat{P})}\right] \beta^{2}} \\
& -\tau \leqslant t \leqslant 0 .
\end{aligned}
$$

In the following, we shall prove that

$$
\eta(t)<\gamma \mathrm{e}^{-\lambda t}+\frac{\epsilon}{\left[b-\frac{a \lambda_{M}\left((G \otimes \Gamma C)^{\mathrm{T}} \hat{P}(G \otimes \Gamma C)\right)}{\beta^{2} \lambda_{m}(\hat{P})}\right] \beta^{2}}, \quad t \geqslant 0 .
$$

If this is not true, according to the estimation (15) and $\eta(t) \in P C\left[[-\tau,+\infty), \mathbf{R}^{+}\right]$, then there obviously exists a $t^{*}>0$ such that

$$
\begin{aligned}
& \eta\left(t^{*}\right) \geqslant \gamma \mathrm{e}^{-\lambda t^{*}}+\frac{\epsilon}{\left[b-\frac{a \lambda_{M}\left((G \otimes \Gamma C)^{\mathrm{T}} \hat{P}(G \otimes \Gamma C)\right)}{\beta^{2} \lambda_{m}(\hat{P})}\right] \beta^{2}} \\
& \eta(t)<\gamma \mathrm{e}^{-\lambda t}+\frac{\epsilon}{\left[b-\frac{a \lambda_{M}\left((G \otimes \Gamma C)^{\mathrm{T}} \hat{P}(G \otimes \Gamma C)\right)}{\beta^{2} \lambda_{m}(\hat{P})}\right] \beta^{2}}, \quad t<t^{*} .
\end{aligned}
$$

Let $\chi=\frac{\epsilon}{\left[b-\frac{a \lambda_{M}\left((G \otimes \Gamma C)^{\mathrm{T}} \hat{P}(G \otimes \Gamma C)\right)}{\beta^{2} \lambda m(\hat{P})}\right] \beta^{2}}$. According to (14) and (18), we get

$$
\begin{aligned}
& \eta\left(t^{*}\right) \leqslant \gamma \leqslant \mathrm{e}^{-b t^{*}}+\int_{0}^{t^{*}} \mathrm{e}^{-b\left(t^{*}-s\right)} \times \\
& {\left[\frac{a \lambda_{M}\left((G \otimes \Gamma C)^{\mathrm{T}} \hat{P}(G \otimes \Gamma C)\right)}{\beta^{2} \lambda_{m}(\hat{P})} \eta(s-\tau(s))+\frac{\epsilon}{\beta^{2}}\right] \mathrm{d} s<} \\
& \mathrm{e}^{-b t^{*}}\left\{\gamma+\chi+\int_{0}^{t^{*}} \mathrm{e}^{b s} \times\right. \\
& {\left[\frac { a \lambda _ { M } ( ( G \otimes \Gamma C ) ^ { \mathrm { T } } \hat { P } ( G \otimes \Gamma C ) ) } { \beta ^ { 2 } \lambda _ { m } ( \hat { P } ) } \left(\gamma \mathrm{e}^{-\lambda(s-\tau(s))}+\right.\right.} \\
& \left.\left.\chi)+\frac{\epsilon}{\beta^{2}}\right] \mathrm{~d} s\right\} \leqslant \\
& \mathrm{e}^{-b t^{*}}\left[\gamma+\chi+\gamma \mathrm{e}^{\lambda \tau} \frac{a \lambda_{M}\left((G \otimes \Gamma C)^{\mathrm{T}} \hat{P}(G \otimes \Gamma C)\right)}{\beta^{2} \lambda_{m}(\hat{P})} \times\right. \\
& \left.\int_{0}^{t^{*}} \mathrm{e}^{(b-\lambda) s} \mathrm{~d} s+b \chi \int_{0}^{t^{*}} \mathrm{e}^{b s} \mathrm{~d} s\right] \leqslant \\
& \mathrm{e}^{-b t^{*}}\left[\gamma+\chi+\gamma(b-\lambda) \int_{0}^{t^{*}} \mathrm{e}^{(b-\lambda) s} \mathrm{~d} s+b \chi \int_{0}^{t^{*}} \mathrm{e}^{b s} \mathrm{~d} s\right]= \\
& \gamma \mathrm{e}^{-\lambda t^{*}}+\frac{\epsilon}{\left[b-\frac{a \lambda_{M}\left((G \otimes \Gamma C)^{\mathrm{T}} \hat{P}(G \otimes \Gamma C)\right)}{\beta^{2} \lambda m(\hat{P})}\right.} .
\end{aligned}
$$

This contradicts (17), so the estimation (16) holds. Letting $\epsilon \rightarrow 0$, we have

$$
0 \leqslant V_{1}(t) \leqslant \eta(t) \leqslant \gamma \mathrm{e}^{-\lambda t}, t \geqslant 0 .
$$

It implies the case 1.

Case 2 can be easily proved in a way similar to case $1 . \square$ Theorem 2. Let Assumption 2 holds. If there exist positive constants $\beta_{k}, \sigma, \xi$ and $\mu$ such that

$$
\begin{aligned}
& \text { 1) }\left\|\hat{Q}\left(I_{n N}+B_{k}\right) \hat{Q}^{-1}\right\| \leqslant \beta_{k} \\
& \text { 2) } \sigma \leqslant \inf \left\{t_{k}-t_{k-1}, \quad k \in \mathbf{N}\right\} \\
& \text { 3) } \sup \left\{\theta_{k}, \quad k \in \mathbf{N}\right\} \leqslant \xi<\mathrm{e}^{\mu \sigma} \\
& \text { 4) } \mu+a+\frac{a \mathrm{e}^{\mu \tau} \lambda_{M}\left((G \otimes \Gamma C)^{\mathrm{T}} \hat{P}(G \otimes \Gamma C)\right)}{\lambda_{m}(\hat{P})}+ \\
& 2 \lambda_{M}\left(-\eta \hat{P}^{-1}+\hat{\Delta}\right) \leqslant 0
\end{aligned}
$$

where $\theta_{k}=\max \left\{1, \beta_{k}^{2}\right\}$, then the network (1) achieves global exponential state synchronization in sense as

$$
\|x(t)\| \leqslant \sqrt{\frac{\lambda_{M}(\hat{P})}{\lambda_{m}(\hat{P})}}\|\Phi\|_{\tau} \mathrm{e}^{-\frac{\mu-\frac{\ln \xi}{\sigma}}{2} t}, \quad t \geqslant 0 .
$$

Proof. According to (12), we can obtain

$$
\begin{aligned}
\dot{V}_{1}(t) \leqslant & {\left[2 \lambda_{M}\left(-\eta \hat{P}^{-1}+\hat{\Delta}\right)+a\right] V_{1}(t)+} \\
& \frac{a \lambda_{M}\left((G \otimes \Gamma C)^{\mathrm{T}} \hat{P}(G \otimes \Gamma C)\right)}{\lambda_{m}(\hat{P})} \times \\
& \left(\sup _{t-\tau \leqslant s \leqslant t} V_{1}(s)\right), t \neq t_{k} .
\end{aligned}
$$

Furthermore,

$$
\begin{aligned}
V_{1}\left(t_{k}\right)= & x^{\mathrm{T}}\left(t_{k}^{-}\right)\left(I_{n N}+B_{k}\right)^{\mathrm{T}} \hat{P}\left(I_{n N}+B_{k}\right) x\left(t_{k}^{-}\right) \leqslant \\
& \left\|\hat{Q}\left(I_{n N}+B_{k}\right) \hat{Q}^{-1}\right\|^{2} x^{\mathrm{T}}\left(t_{k}^{-}\right) \hat{P} x\left(t_{k}^{-}\right) \leqslant \\
& \beta_{k}^{2} V_{1}\left(t_{k}^{-}\right), \quad k \in \mathbf{N} .
\end{aligned}
$$

By Lemma 2, we can get

$$
\begin{aligned}
V_{1}(t) \leqslant & \left(\prod_{0<t_{k} \leqslant t} \theta_{k}\right) \mathrm{e}^{-\mu t}\left(\sup _{-\tau \leqslant s \leqslant 0} \Phi^{\mathrm{T}}(s) \hat{P} \Phi(s)\right) \leqslant \\
& \left(\prod_{0<t_{k} \leqslant t} \theta_{k}\right) \lambda_{M}(\hat{P})\|\Phi\|_{\tau}^{2} \mathrm{e}^{-\mu t}
\end{aligned}
$$

Since $\sigma \leqslant \inf \left\{t_{k}-t_{k-1}, \quad k \in \mathbf{N}\right\}$, one has $k-1 \leqslant \frac{t_{k-1}-t_{0}}{\sigma}=$ $\frac{t_{k-1}}{\sigma}$, which implies

$$
\xi^{k-1} \leqslant \mathrm{e}^{\frac{\ln \xi}{\sigma} t}, \quad t \in\left[t_{k-1}, t_{k}\right), \quad k \in \mathbf{N} .
$$

Then, we have

$$
V_{1}(t) \leqslant \lambda_{M}(\hat{P})\|\Phi\|_{\tau}^{2} \mathrm{e}^{-\left(\mu-\frac{\ln \xi}{\sigma}\right) t} .
$$

Therefore,

$$
\|x(t)\| \leqslant \sqrt{\frac{\lambda_{M}(\hat{P})}{\lambda_{m}(\hat{P})}}\|\Phi\|_{\tau} \mathrm{e}^{-\frac{\mu-\frac{\ln \xi}{\sigma}}{2} t}, \quad t \geqslant 0 .
$$

In the above, two sufficient conditions are given to ensure the global exponential state synchronization of network (1). Next, we shall investigate the output synchronization of complex network (1).

There obviously exist two nonsingular matrices $U \in$ $\mathbf{R}^{q \times q}$ and $H \in \mathbf{R}^{n \times n}$ such that

$$
C=U\left(\begin{array}{cc}
I_{r} & 0 \\
0 & 0
\end{array}\right) H
$$


where $r=\operatorname{rank}(C)$. Let $\hat{x}_{i}(t)=H x_{i}(t)$ and $\hat{y}_{i}(t)=$ $U^{-1} y_{i}(t)$, system (8) can be rewritten as

$$
\left\{\begin{array}{l}
\dot{\hat{x}}_{i}(t)=H f\left(H^{-1} \hat{x}_{i}(t)\right)+a \sum_{j=1}^{N} G_{i j} H \Gamma U \hat{y}_{j}(t-\tau(t)), \\
\quad t \neq t_{k}, \quad t \geqslant 0 \\
\hat{y}_{i}(t)=\left(\begin{array}{cc}
I_{r} & 0 \\
0 & 0
\end{array}\right) \hat{x}_{i}(t) \\
\Delta \hat{x}_{i}=\hat{x}_{i}\left(t_{k}^{+}\right)-\hat{x}_{i}\left(t_{k}^{-}\right)=H B_{i k} H^{-1} \hat{x}_{i}\left(t_{k}^{-}\right), t=t_{k}, \quad k \in \mathbf{N}
\end{array}\right.
$$

where $i=1,2, \cdots, N$. Then, we can get

$$
\left\{\begin{aligned}
\dot{\hat{y}}_{i}(t)= & \hat{f}\left(\hat{x}_{i}(t)\right)+a \sum_{j=1}^{N} G_{i j}\left(\begin{array}{cc}
I_{r} & 0 \\
0 & 0
\end{array}\right) \times \\
& H \Gamma U \hat{y}_{j}(t-\tau(t)), \quad t \neq t_{k}, t \geqslant 0 \\
\Delta \hat{y}_{i}= & \hat{y}_{i}\left(t_{k}^{+}\right)-\hat{y}_{i}\left(t_{k}^{-}\right)=\left(\begin{array}{cc}
I_{r} & 0 \\
0 & 0
\end{array}\right) \times \\
& H B_{i k} H^{-1} \hat{x}_{i}\left(t_{k}^{-}\right), \quad t=t_{k}, k \in \mathbf{N}
\end{aligned}\right.
$$

where $\hat{f}\left(\hat{x}_{i}(t)\right)=\left(\begin{array}{cc}I_{r} & 0 \\ 0 & 0\end{array}\right) H f\left(H^{-1} \hat{x}_{i}(t)\right), i=1,2, \cdots, N$.

In order to obtain our main results, two assumptions are introduced.

Assumption 3. Assume that $\left(\begin{array}{cc}I_{r} & 0\end{array}\right) H B_{i k} H^{-1}=$ $\left(\begin{array}{ll}\hat{B}_{i k} & 0\end{array}\right), \hat{B}_{i k} \in \mathbf{R}^{r \times r}$, and $\hat{f}\left(\hat{x}_{i}(t)\right)=\left(\begin{array}{c}\hat{f}_{1}\left(\hat{x}_{i}^{1}(t)\right) \\ 0\end{array}\right)$ where

$$
\begin{aligned}
& \hat{f}_{1}\left(\hat{x}_{i}^{1}(t)\right) \in \mathbf{R}^{r} \\
& \hat{x}_{i}^{1}(t)=\left(\hat{x}_{i 1}(t), \hat{x}_{i 2}(t), \cdots, \hat{x}_{i r}(t)\right)^{\mathrm{T}} \\
& \hat{x}_{i}^{2}(t)=\left(\hat{x}_{i(r+1)}(t), \hat{x}_{i(r+2)}(t), \cdots, \hat{x}_{i n}(t)\right)^{\mathrm{T}} \\
& \hat{x}_{i}(t)=\left[\left(\hat{x}_{i}^{1}(t)\right)^{\mathrm{T}},\left(\hat{x}_{i}^{2}(t)\right)^{\mathrm{T}}\right]^{\mathrm{T}}, i=1,2, \cdots, N .
\end{aligned}
$$

Assumption 4. There exists positive definite diagonal matrix $Z=\operatorname{diag}\left(z_{1}, z_{2}, \cdots, z_{r}\right)$ and a diagonal matrix $\Psi=\operatorname{diag}\left(\psi_{1}, \psi_{2}, \cdots, \psi_{r}\right)$ such that $\hat{f}_{1}: \mathbf{R}^{r} \rightarrow \mathbf{R}^{r}$ satisfies the following inequality:

$$
x^{\mathrm{T}} Z\left[\hat{f}_{1}(x)-\Psi \boldsymbol{x}\right] \leqslant-\zeta x^{\mathrm{T}} x
$$

for some $\zeta>0$ and all $x \in \mathbf{R}^{r}$.

Remark 1. To the best of our knowledge, this is the first paper to consider the output synchronization of impulsive complex delayed dynamical networks with output coupling, which is a very important and challenging problem. In order to obtain our main results, it is necessary to make the assumption Assumption 3. Practically, Wang and $\mathrm{Wu}^{[27]}$ have studied the output synchronization of complex delayed dynamical networks with output coupling by using this assumption.

According to Assumption 3, we can obtain

$$
\left\{\begin{aligned}
\dot{\hat{x}}_{i}^{1}(t)= & \hat{f}_{1}\left(\hat{x}_{i}^{1}(t)\right)+a \sum_{j=1}^{N} G_{i j} M \hat{x}_{j}^{1}(t-\tau(t)), \\
& t \neq t_{k}, t \geqslant 0 \\
\Delta \hat{x}_{i}^{1}= & \hat{x}_{i}^{1}\left(t_{k}^{+}\right)-\hat{x}_{i}^{1}\left(t_{k}^{-}\right)=\hat{B}_{i k} \hat{x}_{i}^{1}\left(t_{k}^{-}\right) \\
& t=t_{k}, \quad k \in \mathbf{N}
\end{aligned}\right.
$$

where $M=\left(\begin{array}{ll}I_{r} & 0\end{array}\right)_{r \times n} H \Gamma U\left(\begin{array}{c}I_{r} \\ 0\end{array}\right)_{q \times r}, i=1,2, \cdots, N$.
It is clear that the complex network (1) achieves global exponential output synchronization if and only if system (22) is globally exponentially stable.

For convenient analysis, we denote

$$
\begin{aligned}
E & =Z^{\frac{1}{2}}=\operatorname{diag}\left(\sqrt{z_{1}}, \sqrt{z_{2}} \cdots, \sqrt{z_{r}}\right) \\
\hat{Z} & =\operatorname{diag}(Z, Z, \cdots, Z) \\
\hat{E} & =\operatorname{diag}(E, E, \cdots, E) \\
\hat{\Psi} & =\operatorname{diag}(\Psi, \Psi, \cdots, \Psi) \\
\hat{B}_{k} & =\operatorname{diag}\left(\hat{B}_{1 k}, \hat{B}_{2 k}, \cdots, \hat{B}_{N k}\right) \\
y(t) & =\left(y_{1}^{\mathrm{T}}(t), y_{2}^{\mathrm{T}}(t), \cdots, y_{N}^{\mathrm{T}}(t)\right)^{\mathrm{T}} .
\end{aligned}
$$

By the similar proofs of Theorems 1 and 2, we can obtain the following conclusions. Here, we omit their proof to avoid the repetition.

Theorem 3. Let Assumptions 3 and 4 hold.

1) If there exists a positive constant $\beta<1$ such that

$$
\begin{gathered}
\left\|\hat{E}\left(I_{r N}+\hat{B}_{k}\right) \hat{E}^{-1}\right\| \leqslant \beta, \quad k \in \mathbf{N} \\
2 \lambda_{M}\left(-\zeta \hat{Z}^{-1}+\hat{\Psi}\right)+\frac{a \lambda_{M}\left((G \otimes M)^{\mathrm{T}} \hat{Z}(G \otimes M)\right)}{\beta^{2} \lambda_{m}(\hat{Z})}+ \\
a+\frac{2 \ln \beta}{\rho}<0
\end{gathered}
$$

where $\rho=\sup \left\{t_{k}-t_{k-1}, k \in \mathbf{N}\right\}$, then network (1) achieves global exponential output synchronization in the following sense:

$$
\begin{aligned}
\|y(t)\| \leqslant & \beta^{-1} \sqrt{\frac{\lambda_{M}(\hat{Z})}{\lambda_{m}(\hat{Z})}}\left\|I_{N} \otimes U\right\| \times \\
& \left\|I_{N} \otimes\left[\left(\begin{array}{cc}
I_{r} & 0
\end{array}\right) U^{-1}\right]\right\| \times \\
& \|\operatorname{diag}(C, C, \cdots, C) \Phi\|_{\tau} \mathrm{e}^{\frac{-\lambda t}{2}}, t \geqslant 0
\end{aligned}
$$

where $\lambda>0$ is a unique solution of

$$
\begin{aligned}
& \lambda+2 \lambda_{M}\left(-\zeta \hat{Z}^{-1}+\hat{\Psi}\right)+a+\frac{2 \ln \beta}{\rho}+ \\
& \mathrm{e}^{\lambda \tau} \frac{a \lambda_{M}\left((G \otimes M)^{\mathrm{T}} \hat{Z}(G \otimes M)\right)}{\beta^{2} \lambda_{m}(\hat{Z})}=0 .
\end{aligned}
$$

2) If there exists a positive constant $\beta \geqslant 1$ such that

$$
\begin{aligned}
& \left\|\hat{E}\left(I_{r N}+\hat{B}_{k}\right) \hat{E}^{-1}\right\| \leqslant \beta, k \in \mathbf{N} \\
& 2 \lambda_{M}\left(-\zeta \hat{Z}^{-1}+\hat{\Psi}\right)+\frac{a \beta^{2} \lambda_{M}\left((G \otimes M)^{\mathrm{T}} \hat{Z}(G \otimes M)\right)}{\lambda_{m}(\hat{Z})}+ \\
& a+\frac{2 \ln \beta}{\rho}<0
\end{aligned}
$$

where $\rho=\inf \left\{t_{k}-t_{k-1}, k \in \mathbf{N}\right\}$, then network (1) achieves global exponential output synchronization in the following sense:

$$
\begin{gathered}
\|y(t)\| \leqslant \quad \sqrt{\frac{\lambda_{M}(\hat{Z})}{\lambda_{m}(\hat{Z})}}\left\|I_{N} \otimes U\right\|\left\|I_{N} \otimes\left[\left(\begin{array}{cc}
I_{r} & 0
\end{array}\right) U^{-1}\right]\right\| \times \\
\|\operatorname{diag}(C, C, \cdots, C) \Phi\|_{\tau} \mathrm{e}^{\frac{-\lambda t}{2}}, t \geqslant 0
\end{gathered}
$$


where $\lambda>0$ is a unique solution of

$$
\begin{aligned}
\lambda & +2 \lambda_{M}\left(-\zeta \hat{Z}^{-1}+\hat{\Psi}\right)+\frac{2 \ln \beta}{\rho}+ \\
a+\mathrm{e}^{\lambda \tau} \frac{a \beta^{2} \lambda_{M}\left((G \otimes M)^{\mathrm{T}} \hat{Z}(G \otimes M)\right)}{\lambda_{m}(\hat{Z})} & =0 .
\end{aligned}
$$

Theorem 4. Let Assumptions 3 and 4 hold. If there exist positive constants $\beta_{k}, \sigma, \xi$ and $\mu$ such that

$$
\begin{aligned}
& \text { 1) }\left\|\hat{E}\left(I_{r N}+\hat{B}_{k}\right) \hat{E}^{-1}\right\| \leqslant \beta_{k} \\
& \text { 2) } \sigma \leqslant \inf \left\{t_{k}-t_{k-1}, \quad k \in \mathbf{N}\right\} \\
& \text { 3) } \sup \left\{\theta_{k}, \quad k \in \mathbf{N}\right\} \leqslant \xi<\mathrm{e}^{\mu \sigma} \\
& \text { 4) } \mu+\frac{a \mathrm{e}^{\mu \tau} \lambda_{M}\left((G \otimes M)^{\mathrm{T}} \hat{Z}(G \otimes M)\right)}{\lambda_{m}(\hat{Z})}+a+ \\
& 2 \lambda_{M}\left(-\zeta \hat{Z}^{-1}+\hat{\Psi}\right) \leqslant 0
\end{aligned}
$$

where $\theta_{k}=\max \left\{1, \beta_{k}^{2}\right\}$, then network (1) achieves global exponential output synchronization in the following sense:

$$
\begin{aligned}
\|y(t)\| \leqslant & \sqrt{\frac{\lambda_{M}(\hat{Z})}{\lambda_{m}(\hat{Z})}}\left\|I_{N} \otimes U\right\|\left\|I_{N} \otimes\left[\left(\begin{array}{cc}
I_{r} & 0
\end{array}\right) U^{-1}\right]\right\| \times \\
& \|\operatorname{diag}(C, C, \cdots, C) \Phi\|_{\tau} \mathrm{e}^{-\frac{\mu-\frac{\ln \xi}{\sigma}}{2} t}, t \geqslant 0 .
\end{aligned}
$$

Remark 2. To the best of our knowledge, only few researchers have considered the output synchronization for complex networks with time-varying delay and output coupling ${ }^{[27]}$. Wang and $\mathrm{Wu}^{[27]}$ always assumed that the time delay is a differentiable function, and its derivative is less than 1 . The above results remove these conservative restrictions.

\section{Numerical examples}

In this section, two illustrative examples are provided to verify the effectiveness of the proposed theoretical results.

Example 1. Consider a complex network model with 10 nodes, in which each node is a three-dimensional nonlinear system described by

$$
\left(\begin{array}{c}
\dot{x}_{1} \\
\dot{x}_{2} \\
\dot{x}_{3}
\end{array}\right)=\left(\begin{array}{c}
-10 x_{1}+2 x_{2} \\
2 x_{1}-10 x_{2}-x_{1} x_{3} \\
x_{1} x_{2}-6 x_{3}
\end{array}\right)
$$

It is easy to verify that Assumption 2 is satisfied with $\eta=$ $6, P=\operatorname{diag}(1,1,1)$ and $\Delta=\operatorname{diag}(0,0,0)$. Take

$$
\Gamma=\left(\begin{array}{ll}
0.1 & 0.2 \\
0.3 & 0.2 \\
0.2 & 0.1
\end{array}\right), C=\left(\begin{array}{ccc}
0.3 & 0.1 & 0.3 \\
0.1 & 0.1 & 0.3
\end{array}\right)
$$

$a=0.1, \beta_{\omega}=1, B_{i k}=-\frac{1}{2} I_{3}, t_{k}=0.1 k, k \in \mathbf{N}, i=$ $1,2, \cdots, 10$. Matrix $L$ is chosen as

$$
L=\left(\begin{array}{cccccccccc}
-2 & 0 & 0 & 0 & 0 & 0 & 0 & 0 & 1 & 1 \\
0 & -1 & 1 & 0 & 0 & 0 & 0 & 0 & 0 & 0 \\
0 & 1 & -2 & 0 & 0 & 0 & 0 & 0 & 0 & 1 \\
0 & 0 & 0 & -1 & 0 & 0 & 0 & 0 & 0 & 1 \\
0 & 0 & 0 & 0 & -1 & 0 & 0 & 0 & 0 & 1 \\
0 & 0 & 0 & 0 & 0 & -1 & 0 & 0 & 1 & 0 \\
0 & 0 & 0 & 0 & 0 & 0 & -1 & 1 & 0 & 0 \\
0 & 0 & 0 & 0 & 0 & 0 & 1 & -2 & 1 & 0 \\
1 & 0 & 0 & 0 & 0 & 1 & 0 & 1 & -4 & 1 \\
1 & 0 & 1 & 1 & 1 & 0 & 0 & 0 & 1 & -5
\end{array}\right)
$$

We can easily obtain that such complex network is connected, and matrix $G$ is

$$
G=\left(\begin{array}{cccccccccc}
-1 & 0 & 0 & 0 & 0 & 0 & 0 & 0 & \frac{1}{2} & \frac{1}{2} \\
0 & -1 & 1 & 0 & 0 & 0 & 0 & 0 & 0 & 0 \\
0 & \frac{1}{2} & -1 & 0 & 0 & 0 & 0 & 0 & 0 & \frac{1}{2} \\
0 & 0 & 0 & -1 & 0 & 0 & 0 & 0 & 0 & 1 \\
0 & 0 & 0 & 0 & -1 & 0 & 0 & 0 & 0 & 1 \\
0 & 0 & 0 & 0 & 0 & -1 & 0 & 0 & 1 & 0 \\
0 & 0 & 0 & 0 & 0 & 0 & -1 & 1 & 0 & 0 \\
0 & 0 & 0 & 0 & 0 & 0 & \frac{1}{2} & -1 & \frac{1}{2} & 0 \\
\frac{1}{4} & 0 & 0 & 0 & 0 & \frac{1}{4} & 0 & \frac{1}{4} & -1 & \frac{1}{4} \\
\frac{1}{5} & 0 & \frac{1}{5} & \frac{1}{5} & \frac{1}{5} & 0 & 0 & 0 & \frac{1}{5} & -1
\end{array}\right) .
$$

Set $\tau(t)=0.3-0.3 \mathrm{e}^{-t}$. Then, we have $0 \leqslant \tau(t) \leqslant \tau=$ 0.3 , for $t \geqslant 0$. We can find constant $\beta=0.5$ satisfying (2) and (3). According to Theorem 1, we know that network (1) with the above given parameters achieves global exponential state synchronization. The simulation results are shown in Fig. 1.

Example 2. Consider a complex network model with 10 nodes, in which each node is a three-dimensional nonlinear system described by

$$
\left(\begin{array}{c}
\dot{x}_{1} \\
\dot{x}_{2} \\
\dot{x}_{3}
\end{array}\right)=\left(\begin{array}{c}
-5 x_{1}+0.8 x_{2} \\
-0.8 x_{1}-4 x_{2} \\
2 x_{3}+2 x_{1}^{2}+x_{1} x_{2}
\end{array}\right)
$$

Take

$$
\Gamma=\left(\begin{array}{ll}
0.3 & 0.6 \\
0.8 & 0.3 \\
0.9 & 0.2
\end{array}\right), C=\left(\begin{array}{lll}
1 & 0 & 0 \\
0 & 1 & 0
\end{array}\right)
$$

$a=0.2, \beta_{\omega}=1, B_{i k}=\frac{1}{2} I_{3}, t_{k}=k, k \in \mathbf{N}, i=1,2, \cdots, 10$. Matrix $L$ is chosen as 

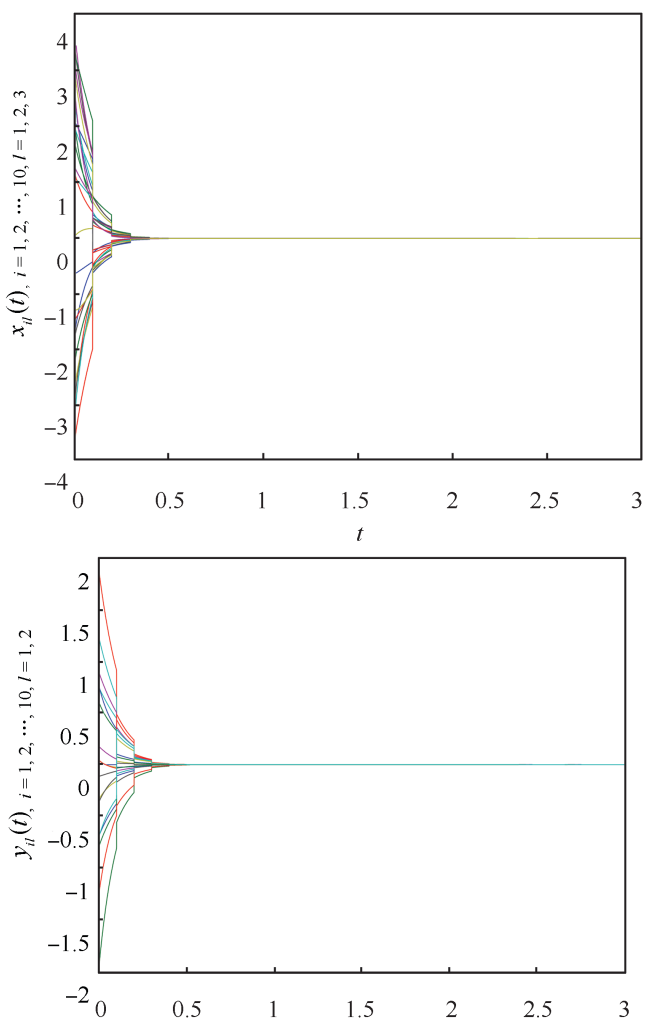

Fig. 1 The change processes of the state variables and output variables

$L=\left(\begin{array}{cccccccccc}-9 & 1 & 1 & 1 & 1 & 1 & 1 & 1 & 1 & 1 \\ 1 & -1 & 0 & 0 & 0 & 0 & 0 & 0 & 0 & 0 \\ 1 & 0 & -1 & 0 & 0 & 0 & 0 & 0 & 0 & 0 \\ 1 & 0 & 0 & -1 & 0 & 0 & 0 & 0 & 0 & 0 \\ 1 & 0 & 0 & 0 & -1 & 0 & 0 & 0 & 0 & 0 \\ 1 & 0 & 0 & 0 & 0 & -1 & 0 & 0 & 0 & 0 \\ 1 & 0 & 0 & 0 & 0 & 0 & -1 & 0 & 0 & 0 \\ 1 & 0 & 0 & 0 & 0 & 0 & 0 & -2 & 1 & 0 \\ 1 & 0 & 0 & 0 & 0 & 0 & 0 & 1 & -2 & 0 \\ 1 & 0 & 0 & 0 & 0 & 0 & 0 & 0 & 0 & -1\end{array}\right)$.

We can easily obtain that such complex network is connected, and matrix $G$ is

$G=\left(\begin{array}{cccccccccc}-1 & \frac{1}{9} & \frac{1}{9} & \frac{1}{9} & \frac{1}{9} & \frac{1}{9} & \frac{1}{9} & \frac{1}{9} & \frac{1}{9} & \frac{1}{9} \\ 1 & -1 & 0 & 0 & 0 & 0 & 0 & 0 & 0 & 0 \\ 1 & 0 & -1 & 0 & 0 & 0 & 0 & 0 & 0 & 0 \\ 1 & 0 & 0 & -1 & 0 & 0 & 0 & 0 & 0 & 0 \\ 1 & 0 & 0 & 0 & -1 & 0 & 0 & 0 & 0 & 0 \\ 1 & 0 & 0 & 0 & 0 & -1 & 0 & 0 & 0 & 0 \\ 1 & 0 & 0 & 0 & 0 & 0 & -1 & 0 & 0 & 0 \\ \frac{1}{2} & 0 & 0 & 0 & 0 & 0 & 0 & -1 & \frac{1}{2} & 0 \\ \frac{1}{2} & 0 & 0 & 0 & 0 & 0 & 0 & \frac{1}{2} & -1 & 0 \\ 1 & 0 & 0 & 0 & 0 & 0 & 0 & 0 & 0 & -1\end{array}\right)$.

It is obvious that we can take $U=\left(\begin{array}{ll}1 & 0 \\ 0 & 1\end{array}\right), H=$ $\left(\begin{array}{lll}1 & 0 & 0 \\ 0 & 1 & 0 \\ 0 & 0 & 1\end{array}\right)$. Moreover, $M=\left(\begin{array}{cc}0.3 & 0.6 \\ 0.8 & 0.3\end{array}\right), \quad \hat{B}_{i k}=$ $\frac{1}{2} I_{2}, \hat{f}_{1}\left(\hat{x}_{i}^{1}(t)\right)=\left(\begin{array}{cc}-5 & 0.8 \\ -0.8 & -4\end{array}\right) \hat{x}_{i}^{1}(t), \hat{x}_{i}^{1}(t) \in \mathbf{R}^{2}, i=$ $1,2, \cdots, 10$. It is easy to verify that Assumption 4 is satisfied with $\zeta=4, Z=\operatorname{diag}(1,1)$ and $\Psi=\operatorname{diag}(0,0)$.

Set $\tau(t)=0.4-0.4 \mathrm{e}^{-t}$. Then, we have $0 \leqslant \tau(t) \leqslant \tau=$ 0.4. We can find constants $\beta_{k}=1.5, \mu=2, \xi=2.5, \sigma=1$ satisfying Theorem 4. Therefore, we know that network (1) with the above given parameters achieves global exponential output synchronization. The simulation results are shown in Fig. 2.
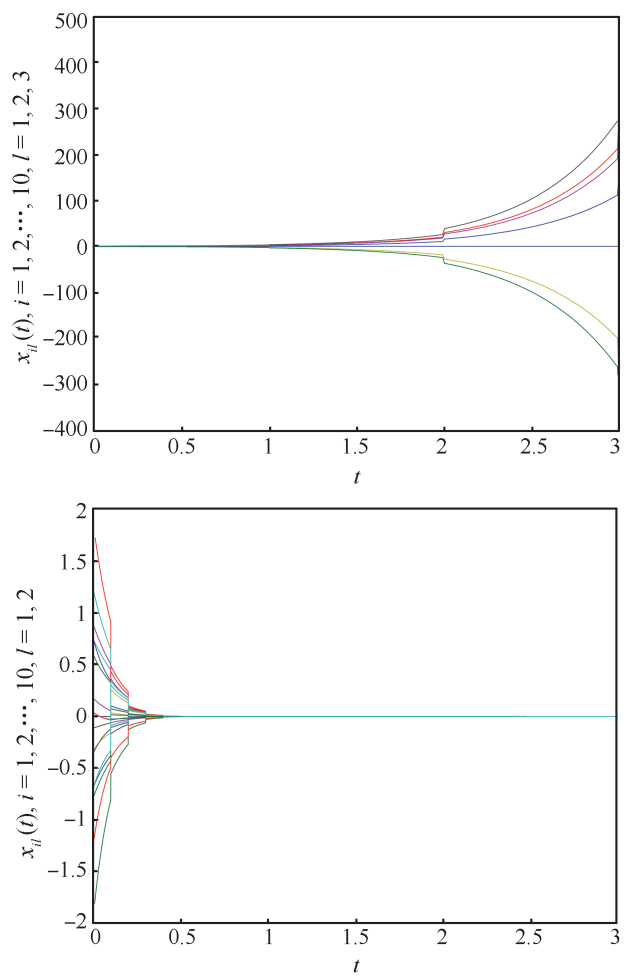

Fig. 2 The evolution of the state variables and output variables

Remark 3. According to the evolution of the state variables and output variables in Fig. 1, we can see dearly that the complex network realizes state synchronization and output synchronization. However, the outputs are synchronous but the states are asynchronous in Fig. 2. The main reason is that output variables are only related to part of states. In other words, only part of states are needed to make output synchronization to come true. Therefore, it is important and interesting to study the output synchronization of the complex networks.

\section{Conclusions}

A new impulsive complex delayed dynamical network model with output coupling has first been introduced. We have considered the state synchronization and output synchronization of the proposed network model. Some global exponential state synchronization and output synchronization criteria have been established by employing impulsive 
delay differential inequalities. Illustrative simulations have been provided to verify the correctness and effectiveness of the obtained results.

\section{Acknowledgement}

The authors would like to thank the associate editors and anonymous reviewers for their valuable comments and suggestions.

\section{References}

[1] J. L. Wang, Z. C. Yang, H. N. Wu. Passivity analysis of complex dynamical networks with multiple time-varying delays. Journal of Engineering Mathematics, vol. 74, no. 1, pp. 175$188,2012$.

[2] J. L. Wang, H. N. Wu, Z. C. Yang. Passivity analysis of impulsive complex networks. International Journal of $\mathrm{Au}$ tomation and Computing, vol. 8, no. 4, pp. 484-489, 2011.

[3] J. L. Wang, Z. C. Yang, T. W. Huang, M. Q. Xiao. Synchronization criteria in complex dynamical networks with nonsymmetric coupling and multiple time-varying delays. Applicable Analysis, vol.91, no. 5, pp. 923-935, 2012.

[4] J. L. Wang, Z. C. Yang, T. W. Huang, M. Q. Xiao. Local and global exponential synchronization of complex delayed dynamical networks with general topology. Discrete and Continuous Dynamical Systems - Series B, vol.16, no.1, pp. 393-408, 2011.

[5] J. L. Wang, H. N. Wu. Stability analysis of impulsive parabolic complex networks. Chaos, Solitons \& Fractals, vol. 44, no. 11, pp. 1020-1034, 2011.

[6] J. L. Wang, H. N. Wu. Synchronization criteria for impulsive complex dynamical networks with time-varying delay. Nonlinear Dynamics, vol. 70, no. 1, pp. 13-24, 2012.

[7] Y. Dai, Y. Z. Cai, X. M. Xu. Synchronization and exponential estimates of complex networks with mixed time-varying coupling delays. International Journal of Automation and Computing, vol. 6, no. 3, pp. 301-307, 2009.

[8] W. W. Yu, J. D. Cao, J. H. Lü. Global synchronization of linearly hybrid coupled networks with time-varying delay. SIAM Journal on Applied Dynamical Systems, vol. 7, no. 1, pp. 108-133, 2008.

[9] J. Q. Lu, J. Kurths, J. D. Cao, N. Mahdavi, C. Huang. Synchronization control for nonlinear stochastic dynamical networks: Pinning impulsive strategy. IEEE Transactions on Neural Networks and Learning Systems, vol.23, no. 2, pp. 285-292, 2012.

[10] J. Q. Lu, D. W. C. Ho, J. D. Cao, J. Kurths. Exponential synchronization of linearly coupled neural networks with impulsive disturbances. IEEE Transactions on Neural Networks, vol. 22, no. 2, pp. 329-336, 2011.

[11] C. G. Li, G. R. Chen. Synchronization in general complex dynamical networks with coupling delays. Physica A: Statistical Mechanics and its Applications, vol.343, pp. 263278, 2004.

[12] L. Y. Xiang, Z. Q. Chen, Z. X. Liu, F. Chen, Z. Z. Yuan. Pinning control of complex dynamical networks with heterogeneous delays. Computers \& Mathematics with Applications, vol. 56, no. 5, pp. 1423-1433, 2008.
[13] Q. Y. Wang, Z. S. Duan, G. R. Chen, Z. S. Feng. Synchronization in a class of weighted complex networks with coupling delays. Physica A: Statistical Mechanics and its Applications, vol. 387, no. 22, pp. 5616-5622, 2008.

[14] Q. J. Zhang, J. A, Lu, J. H. Lü, C. K. Tse. Adaptive feedback synchronization of a general complex dynamical network with delayed nodes. IEEE Transactions on Circuits and Systems II: Express Briefs, vol. 55, no. 2, pp. 183-187, 2008.

[15] X. Q. Wu. Synchronization-based topology identification of weighted general complex dynamical networks with timevarying coupling delay. Physica A: Statistical Mechanics and its Applications, vol. 387, no. 4, pp.997-1008, 2008.

[16] L. Wang, H. P. Dai, H. Dong, Y. H. Shen, Y. X. Sun. Adaptive synchronization of weighted complex dynamical networks with coupling time-varying delays. Physics Letters $A$, vol. 372, no. 20, pp. 3632-3639, 2008.

[17] S. M. Cai, J. Zhou, L. Xiang, Z. R. Liu. Robust impulsive synchronization of complex delayed dynamical networks. Physics Letters A, vol. 372, no. 30, pp. 4990-4995, 2008.

[18] L. Y. Xiang, Z. X. Liu, Z. Q. Chen, Z. Z. Yuan. Pinning weighted complex networks with heterogeneous delays by a small number of feedback controllers. Science in China Series F: Information Sciences, vol.51, no. 5, pp.511-523, 2008.

[19] K. Li, C. H. Lai. Adaptive-impulsive synchronization of uncertain complex dynamical networks. Physics Letters A, vol. 372, no. 10, pp. 1601-1606, 2008.

[20] W. W. Lin, Y. Q. Wang. Chaotic synchronization in coupled map lattices with periodic boundary conditions. SIAM Journal on Applied Dynamical Systems, vol. 1, no. 2, pp. 175-189, 2002.

[21] J. Zhou, J. A. Lu, J. H. Lü. Pinning adaptive synchronization of a general complex dynamical network. Automatica, vol. 44 , no. 4, pp. 996-1003, 2008.

[22] Z. Li, L. C. Jiao, J. J. Lee. Robust adaptive global synchronization of complex dynamical networks by adjusting timevarying coupling strength. Physica A: Statistical Mechanics and its Applications, vol. 387, no. 5-6, pp. 1369-1380, 2008.

[23] P. L. Lu, Y. Yang, L. Huang. Synchronization of linearly coupled networks of deterministic ratchets. Physics Letters A, vol. 372, no. 22, pp. 3978-3985, 2008.

[24] W. L. Lu, F. M. Atay, J. Jost. Synchronization of discretetime dynamical networks with time-varying coupling. SIAM Journal on Mathematical Analysis, vol. 39, no. 4, pp. 12311259, 2007.

[25] S. Wen, S. H. Chen, W. L. Guo. Adaptive global synchronization of a general complex dynamical network with nondelayed and delayed coupling. Physics Letters A, vol. 372, no. 42 , pp. $6340-6346,2008$.

[26] G. P. Jiang, W. K. S. Tang, G. R. Chen. A state-observerbased approach for synchronization in complex dynamical networks. IEEE Transactions on Circuits and Systems I: Regular Papers, vol. 53, no. 12, pp. 2739-2745, 2006.

[27] J. L. Wang, H. N. Wu. Local and global exponential output synchronization of complex delayed dynamical networks. Nonlinear Dynamics, vol. 67, no. 1, pp. 497-504, 2012.

[28] S. J. Long, D. Y. Xu. Delay-dependent stability analysis for impulsive neural networks with time varying delays. Neurocomputing, vol. 71, no. 7-9, pp. 1705-1713, 2008. 
[29] Z. C. Yang, D. Y. Xu. Stability analysis and design of impulsive control systems with time delay. IEEE Transactions on Automatic Control, vol. 52, no. 8, pp. 1448-1454, 2007.

[30] Z. C. Yang, D. Y. Xu. Stability analysis of delay neural networks with impulsive effects. IEEE Transactions on Circuits Systems II: Express Briefs, vol.52, no. 8, pp. 517-521, 2005.

[31] Z. C. Yang, D. Y. Xu. Impulsive effects on stability of Cohen-Grossberg neural networks with variable delays. Applied Mathematics and Computation, vol. 177, no. 1, pp. 63$78,2006$.

[32] H. G. Zhang, T. D. Ma, G. B. Huang, Z. L. Wang. Robust global exponential synchronization of uncertain chaotic delayed neural networks via dual-stage impulsive control. IEEE Transactions on Systems, Man, Cybernetics, Part B: Cybernetics, vol. 40, no. 3, pp. 831-844, 2010.

[33] J. Zhou, L. Xiang, Z. R. Liu. Synchronization in complex delayed dynamical networks with impulsive effects. Physica A: Statistical Mechanics and its Applications, vol. 384, no. 2, pp. 684-692, 2007.

[34] S. Zheng, G. G. Dong, Q. S. Bi. Impulsive synchronization of complex networks with non-delayed and delayed coupling. Physics Letters A, vol.373, no. 46, pp. 4255-4259, 2009 .

[35] Y. Q. Yang, J. D. Cao. Exponential synchronization of the complex dynamical networks with a coupling delay and impulsive effects. Nonlinear Analysis: Real World Applications, vol.11, no. 3, pp. 1650-1659, 2010.

[36] J. Q. Lu, D. W. C. Ho, J. D. Cao. A unified synchronization criterion for impulsive dynamical networks. Automatica, vol. 46, no. 7, pp. 1215-1221, 2010.

[37] W. L. Lu, T. P. Chen. New approach to synchronization analysis of linearly coupled ordinary differential systems. Physica D: Nonlinear Phenomena, vol. 213, no. 2, pp. 214$230,2006$.

[38] W. L. Guo, F. Austin, S. H. Chen. Global synchronization of nonlinearly coupled complex networks with non-delayed and delayed coupling. Communications in Nonlinear Science and Numerical Simulation, vol.15, no.6, pp.16311639, 2010.

[39] V. Kolmanovskii, A. Myshkis. Introduction to the Theory and Applications of Functional Differential Equations. London: Kluwer Academic, 1999.

[40] V. Lakshmikantham, D. D. Bainov, P. S. Simeonov. Theory of Impulsive Differential Equations, Singapore: World Scientific, 1989.

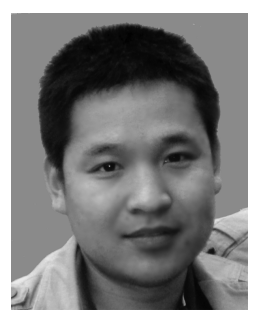

ing author)

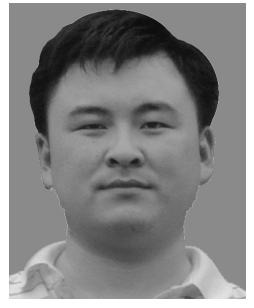

Jin-Liang Wang received the M.Sc. degree in applied mathematics from Department of Mathematics, Chongqing Normal University, China in 2010. Currently, he is a $\mathrm{Ph}$. D. candidate at School of Automation Science and Electrical Engineering, Beihang University, China. He serves as a reviewer for several journals. He is the recipient of 2011 Excellent Master Degree Thesis Award of Chongqing City.

His research interests include complex networks, multi-agent systems, distributed parameter systems, impulsive control systems.

E-mail: wangjinliang1984@tom.com 